\title{
Predictive factors of cough after uniportal video-assisted thoracoscopic pulmonary resection
}

\author{
Liu-Ying Pan ${ }^{1,2 \#}$, Li-Ping Peng ${ }^{1,2 \#}$, Chun $\mathrm{Xu}^{1,2}$, Cheng Ding ${ }^{1,2}$, Jun Chen $^{1,2}$, Wen-Yi Wang ${ }^{1,2}$, \\ Xin-Yu Zhu ${ }^{1,2}$, Jun Zhao ${ }^{1,2}$, Chang $\mathrm{Li}^{1,2}$ \\ ${ }^{1}$ Department of Thoracic Surgery, The First Affiliated Hospital of Soochow University, Medical College of Soochow University, Suzhou, China; \\ ${ }^{2}$ Institute of Thoracic Surgery, The First Affiliated Hospital of Soochow University, Suzhou, China \\ Contributions: (I) Conception and design: LY Pan, LP Peng, J Zhao, C Li; (II) Administrative support: J Zhao, C Li; (III) Provision of study \\ materials or patients: J Zhao, C Xu, C Ding, C Li; (IV) Collection and assembly of data: LY Pan, LP Peng, XY Zhu, J Chen; (V) Data analysis and \\ interpretation: LY Pan, LP Peng, C Li; (VI) Manuscript writing: All authors; (VII) Final approval of manuscript: All authors. \\ \#These authors contributed equally to this work. \\ Correspondence to: Jun Zhao; Chang Li. Department of Thoracic Surgery, The First Affiliated Hospital of Soochow University, Medical College of \\ Soochow University, Suzhou 215000, China. Email: zhaojia0327@126.com; cli@suda.edu.cn.
}

Background: Cough is one of the shared complications after lung surgery. In this study, a prospective analysis was conducted for exploring the risk factors of persistent cough after uniportal video-assisted thoracoscopic pulmonary resection.

Methods: One hundred thirty-five patients with pulmonary nodules who underwent surgical treatment in the same surgical group from November 2019 to January 2020 were enrolled in this prospective study. The severity of cough and its impact on patients' quality of life before and after surgery were assessed by the Mandarin Chinese version of the Leicester cough questionnaire (LCQ-MC), and postoperative cough was tested by the cough visual analog scale (VAS) and cough symptom score (CSS). Risk factors of cough after pulmonary resection (CAP) were determined by univariate and multivariate logistic regression analysis.

Results: The incidence of postoperative cough was $24.4 \%$ (33 of 135 patients). Univariate analysis showed that gender (female), the surgical site (upper right), the resection (lobectomy), subcarinal lymph node dissection, postoperative acid reflux, length of hospitalization contributed to the development of CAP resection. Multivariate logistic regression analysis showed that the resection (lobectomy) (OR 3.590, 95\% CI: 0.637-20.300, $\mathrm{P}=0.017$ ), subcarinal lymph node dissection (OR 4.420, 95\% CI: 1.342-14.554, P=0.001), postoperative acid reflux (OR 13.55, 95\% CI: 3.186-57.633, $\mathrm{P}<0.001$ ) and duration of anesthesia (over 153 minutes, OR 0.987, 95\% CI: 0.978-0.997, $\mathrm{P}=0.011$ ) were independent risk factors for postoperative cough.

Conclusions: The application of uniportal video-assisted thoracoscopic techniques to several types of lung surgery are conducive to enhanced recovery after surgery (ERAS). Postoperative cough is related to an ocean of factors, the resection (lobectomy), subcarinal lymph node dissection, postoperative acid reflux, and duration of anesthesia (over 153 minutes) are independent high-risk factors for CAP resection.

Trial registration: This study was registered on ClinicalTrials.gov (NCT04204148).

Keywords: Cough after pulmonary resection (CAP); uniportal video-assisted thoracoscopic surgery (uniportal VATS); enhanced recovery after surgery (ERAS); risk factor

Submitted Jul 24, 2020. Accepted for publication Sep 24, 2020.

doi: $10.21037 /$ jtd-20-2652

View this article at: http://dx.doi.org/10.21037/jtd-20-2652

(C) Journal of Thoracic Disease. All rights reserved. 


\section{Introduction}

The extensive application of low-dose chest helical computed tomography (CT) screening in clinic practice arises with the rapid development of medical technology, enabling the early diagnosis of pulmonary nodules in many patients. Pulmonary nodules include malignant tumors, benign tumors, lung infections, interstitial diseases, etc. Surgical resection is the primary treatment for resectable non-small cell lung cancer (NSCLC) and benign lung tumors. Shared complications after pulmonary resection are arrhythmia, pulmonary infections, postoperative cough, postoperative pneumothorax, chylothorax, pulmonary embolism, etc. Among them, persistent cough after pulmonary resection (CAP) is one of the most shared complications following thoracic pulmonary resection (1). CAP refers to a dry cough that lasts no less than two weeks after pulmonary resection, except for nasal drip syndrome, bronchial asthma, or oral angiotensin-converting enzyme inhibitor (ACEI) drugs, the chest X-ray revealed no apparent abnormality (2). Previous studies have reported the incidence of CAP can reach $25-50 \%$, and the probabilities of coughing within one year and five years after surgery are over $50 \%$ and $18 \%$, respectively (3). The postoperative cough can last a long time. Patients with a milder cough suffer more pain from the incision, have a more extended hospital stay, cost more, and are prone to unfavorable postoperative recovery (4). In more severe cases, postoperative cough will lead to organ damage and psychological and social dysfunction. It brings about symptoms including vomiting, chest pain, and rib fractures, affects interpersonal relationships, interferes with lifestyles, makes people feel anxious and depressed, etc., exerting a far-reaching adverse effect on patients' quality of life $(5,6)$.

Hence, adequate attention should be paid to the occurrence of persistent CAP. Previous studies have established that the mechanism of coughing is associated with activation of the lung C-fiber and vagus nerve $(7,8)$. Huang et al. (9) showed the cavity left after mediastinal lymph node resection stimulated cough receptors and caused the cough reflex once it was subjected to mechanical or chemical irritations. Lin et al. (10) manifested that females, lower paratracheal node resection, and subcarinal node resection were independent risk factors for postoperative cough. A retrospective study by Chen et al. (11) revealed that spontaneous respiration thoracic anesthesia could reduce the incidence of postoperative cough, speed up postoperative recovery, and improve the quality of life of patients. Lai et al. (12) analyzed the demographic characteristics of 1,882 Chinese chronic cough patients and explored the relationship of their age and sex distribution with cough reflex sensitivity. This study suggested that chronic cough patients in China had the same gender distribution and were middle-aged. Kavalcikova-Bogdanova and his colleagues (13) showed that female patients might be more prone to chronic cough since female hormones could regulate neural excitability, laryngeal hypersensitivity, and the mast cell function. The mechanism and influencing factors of CAP are still controversial.

Most of the previous research on CAP was retrospective, mainly focused on thoracotomy and multiport videoassisted thoracoscopic surgery (VATS), with no effort made on standard methods for assessing postoperative cough $(2,9)$. The advancement of science and technology and the improvement of thoracic surgeons' operation skills are accompanied by the increasingly full application of uniportal VATS (14). Compared with traditional thoracotomy and multiport VATS, uniportal VATS can reduce postoperative complications, improve the quality of postoperative life, shorten postoperative hospitalization time, and promote faster postoperative recovery (15) - this prospective study related to uniportal VATS. The Mandarin Chinese version of the Leicester cough questionnaire (LCQ-MC), cough visual analog scale (VAS), cough symptom score (CSS) was used to test cough. The factors related to the occurrence of CAP were analyzed in this study to further enrich the theory of enhanced recovery after surgery (ERAS) in thoracic surgery.

We present the following article in accordance with the STROBE reporting checklist (available at http://dx.doi. org/10.21037/jtd-20-2652).

\section{Methods}

This prospective non-intervention observational study was conducted in the same surgery group of the Department of Thoracic Surgery of The First Affiliated Hospital of Soochow University. The study protocol was approved by the Ethics Committee of The First Affiliated Hospital of Soochow University, Suzhou, China, and was registered on ClinicalTrials.gov (NCT04204148). All patients have signed the written informed consent before the operation. The study was conducted in accordance with the Declaration of Helsinki (as revised in 2013). 


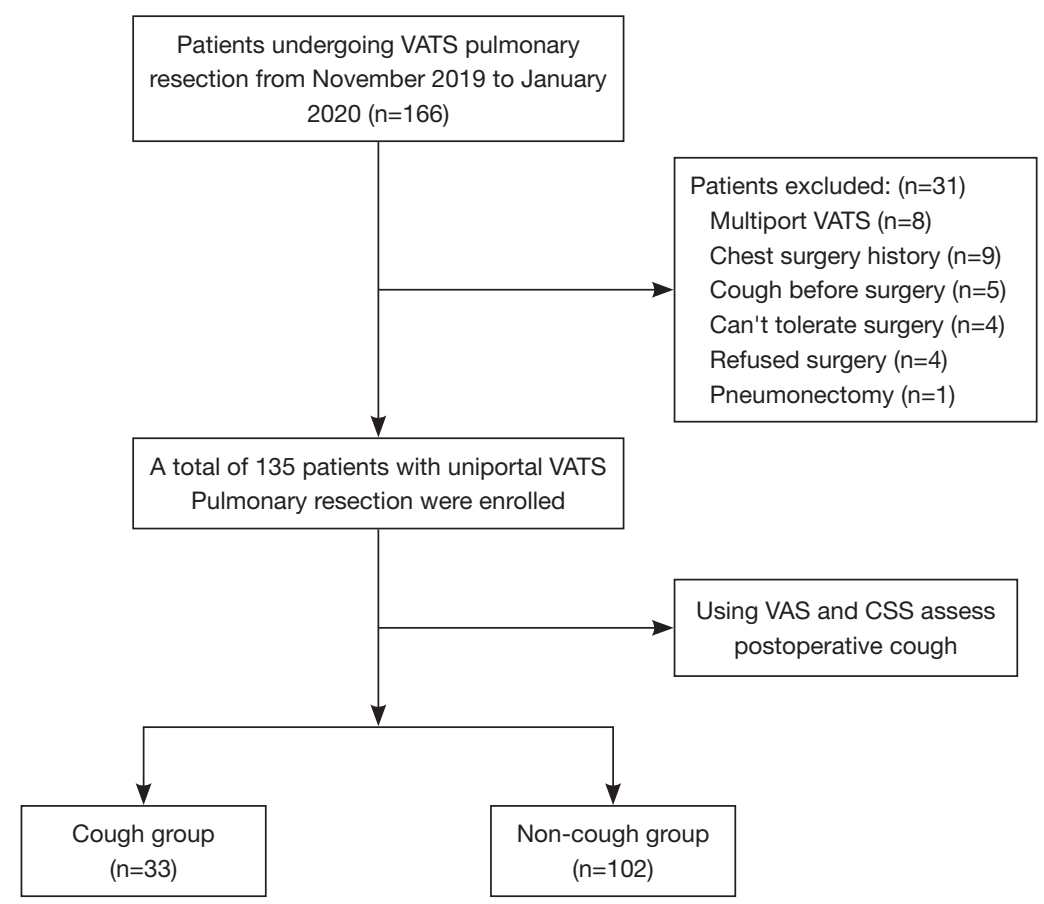

Figure 1 Patient flowchart. VATS, video-assisted thoracoscopic surgery; VAS, visual analog scale; CSS, cough symptoms score.

\section{Patient selection}

One hundred and thirty-five patients who underwent resection of pulmonary nodules from November 2019 to January 2020 were enrolled in this prospective study. They all met the following inclusion criteria: (I) males and females, with an age of over 18; (II) uniportal VATS; (III) no cough symptoms within two weeks before surgery; (IV) undergoing pulmonary wedge resection, segmentectomy or lobectomy; (V) signing the informed consent before surgery. Patients who (I) could not tolerate surgery because of cardiopulmonary and other organ dysfunction; (II) changed to multiport VATS or thoracotomy from uniportal VATS; (III) showed pneumonia by chest radiographs or CT in recent months; (IV) had distant metastasis; (V) coughed before surgery because of respiratory infectious diseases, pharyngitis, allergic rhinitis, nasal drip syndrome, etc.; (VI) suffered severe postoperative complications, including severe infections, significant nerve injury, pulmonary embolism, chylothorax, etc., and (VII) refused to be followed up were excluded (Figure 1).

\section{Surgical techniques}

The patient was placed in the healthy lateral position and underwent double-lumen endobronchial tube (DLT) intubation and the healthy side one-lung ventilation under general anesthesia during surgery. A single small incision with a size of $3-5 \mathrm{~cm}$ was performed in all patients at the fourth or fifth intercostal space in the anterior axillary line. Determined by preoperative image data and intra-operative rapid pathological results, lobectomy and sublobular resection, including wedge resection and segmentectomy, were taken as the surgical methods. Lymph nodes were grouped by the American Joint Committee on Cancer (AJCC) criteria (16) and were resected or sampled according to the Chinese Guidelines for the Diagnosis and Treatment of Primary Lung Cancer (2018) (17). A 28-F chest drainage tube was placed at the surgical port after surgery.

\section{Postoperative management}

All patients were sent back to the thoracic surgery unit after the surgery. Professional nurses recorded their postoperative vital signs, 24-hour drainage, urine volume, rehydration volume, etc. Patients were guided to cough and expectorate correctly and encouraged to get out of bed early after surgery. The chest radiograph, blood routine, and electrolytes were taken on the first day after surgery. Patients 
whose 24-hour chest drainage was less than $200 \mathrm{~mL}$, a chest radiograph showed no pneumothorax, and a chest tube had no air leakage allowed to remove their chest tube.

\section{Evaluation methods for postoperative acid reflux}

The reflux diagnostic questionnaire (RDQ) was used to conduct a questionnaire survey on the frequency and severity of reflux symptoms in all postoperative patients included in this study. Symptoms of reflux include acid reflux, pain behind the breastbone, regurgitation, and heartburn. The two scores are added together as the RDQ score, and the score is $>12$ points as the diagnostic threshold (18).

\section{Evaluation methods and grouping criteria for coughing}

The LCQ-MC is a useful tool to assess the severity of cough and its impact on patients' quality of life. It is more flexible than other cough-related questionnaires and has practical value in the clinical evaluation and diagnosis of cough (19). The LCQ-MC comprised physical, psychological, and social dimensions, including eight physiological items, seven psychological items, and four social items, totaling 19 multiple-choice questions, seven options for each question (forward scoring, 1-7 grades; a higher score shows a lighter cough). The score of each dimension takes the average of the scores of all items in this dimension (1-7). The total score is the sum of the average scores of all three dimensions (3-21). All the patients enrolled in this study completed the LCQ-MC one day before surgery, one month after surgery, and three months after surgery under the guidance of two trained thoracic medical staff.

The postoperative cough was evaluated using VAS and CSS (20). The VAS is a linear scoring method, with a scale of $0-100 \mathrm{~mm}$ (0 means no cough, and 100 stands for the most severe cough). Patients were needed to mark the severity of the cough on the scale according to their feeling of cough. The distance between the marked point and the starting point measured was taken as the score. A higher score suggested a more severe cough. The CSS includes daytime and nighttime CSSs. The score of each choice ranges from 0 to 3 points from the severity. The scoring system reflects the frequency and intensity of coughing and its impact on the quality of life. Patients with the VAS reaching $60 \mathrm{~mm}$ and the CSS over 2 points were classified into the cough group.

\section{Statistical analysis}

Data analysis was performed using SPSS 25.0 software (Statistical Package for the Social Sciences, Chicago, IL, USA). The count data were expressed in terms of frequency and percentage (\%). A standard chi-square test was conducted to compare distinct groups. Measurement data accorded with normal distribution were analyzed by an independent sample $T$-test and represented by mean \pm standard deviation; Measurement data not conforming to normal distribution were analyzed by the Wilcoxon ranksum test and represented by median and 25th (Q1) and 75th (Q3) quantiles. Multivariate logistic regression analysis was conducted to explore independent risk factors of CAP, and the receiver operating characteristic (ROC) curve was used to determine the cutoff value of each variable. $\mathrm{P}$ value of $<0.05$ showed statistical significance.

\section{Results}

\section{Patients' clinic and pathological data}

Altogether, 135 patients undergoing surgical treatment for pulmonary nodules from November 1, 2019, to January 31, 2020, were enrolled in this study, including 58 males and 77 females. These patients were divided into the cough group (33 cases) and the non-cough group (102 cases), with the incidence of $24.4 \%$. Lung wedge resection was performed on 68 patients, lobectomy on 55 patients, segmentectomy on 12 patients. Adenocarcinoma in situ (AIS) was observed in 56 patients and adenocarcinoma in 52 patients, 27 patients were detected with another pathological type. The general clinical data of patients are shown in Table 1.

\section{LCQ-MC score before and after surgery}

All patients were followed up for three months after surgery, and 33 of them developed a cough. There was no statistical difference $(\mathrm{P}>0.05)$ in the LCQ-MC score between the cough group and the non-cough group before surgery. The LCQ-MC score of the cough group was significantly lower than the non-cough group at one month and three months after the surgery $(\mathrm{P}<0.05)$. In the cough group, the LCQ-MC score at three months after operation $(17.34 \pm 0.70)$ was higher than before operation $(16.77 \pm 0.81)$ and that at one month after operation $(16.60 \pm 1.02)$, as shown in Table 2. 
Table 1 The clinical characteristics and univariate analysis of patients with and without postoperative cough after pulmonary resection

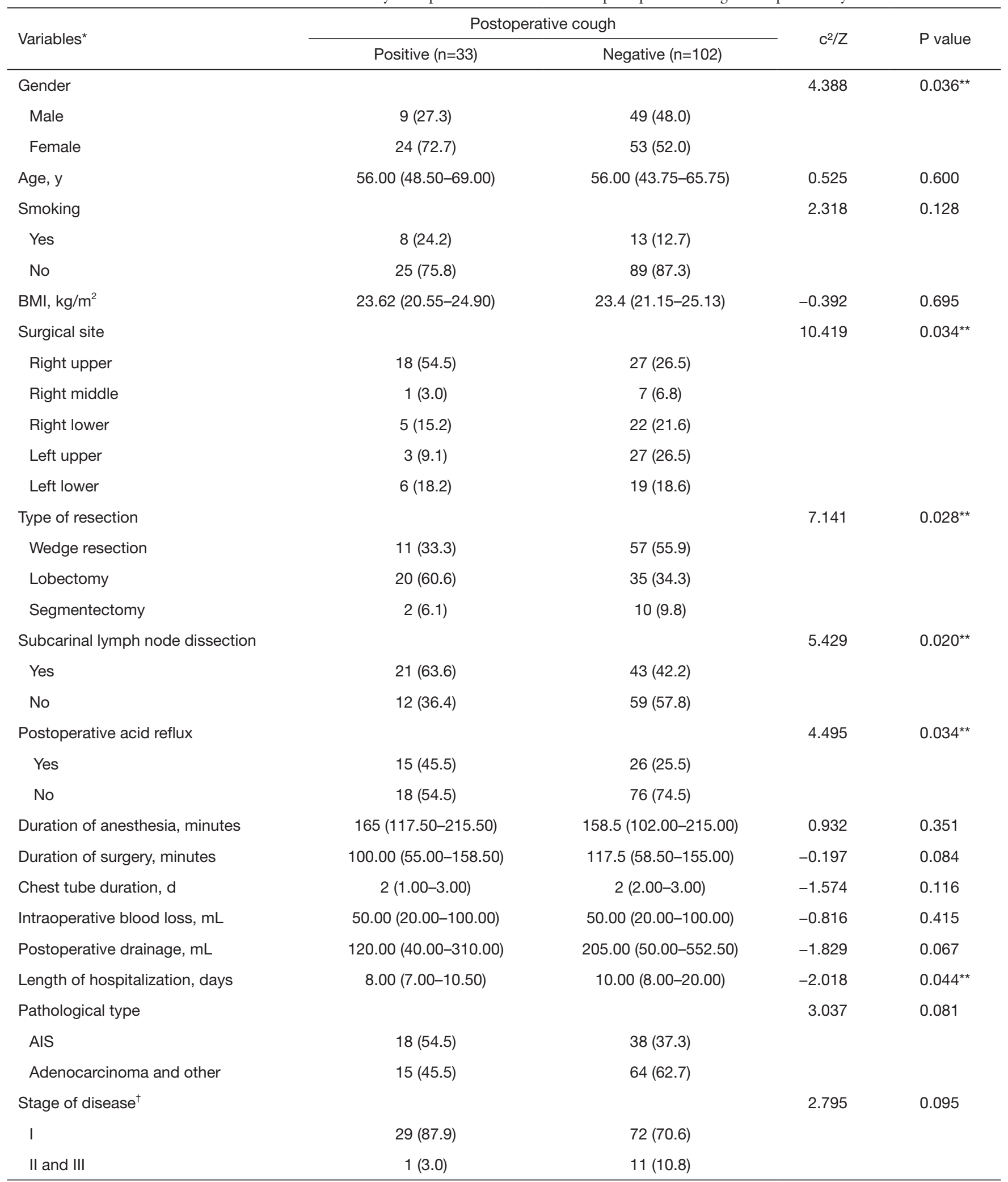

${ }^{*}$, continuous data are shown as median $(\mathrm{Q} 1-\mathrm{Q} 3)$ and categoric data as $\mathrm{n}(\%) ;{ }^{* *}$, statistically significant $(\mathrm{P}<0.05) ;{ }^{\dagger}$, exclude $(\mathrm{n}=22$, benign lesion). BMI, body mass index; AIS, adenocarcinoma in situ. 
Table 2 The mean LCQ-MC score before and after surgery

\begin{tabular}{lccc}
\hline \multirow{2}{*}{ Variables* $^{*}$} & \multicolumn{2}{c}{ LCQ-MC score } & \multirow{2}{*}{$\mathrm{P}$ value } \\
\cline { 2 - 3 } & Cough & Non-cough & 1.426 \\
\hline Preoperative & $16.77 \pm 0.81$ & $17.42 \pm 0.98$ & 0.183 \\
One month after surgery & $16.60 \pm 1.02$ & $17.64 \pm 0.77$ & 6.198 \\
Three months after surgery & $17.34 \pm 0.70$ & $17.89 \pm 0.72$ & 3.868 \\
\hline
\end{tabular}

${ }^{*}$, continuous data are shown as mean $\pm \mathrm{SD}$; ${ }^{*}$, statistically significant $(\mathrm{P}<0.05)$. LCQ-MC, The Mandarin Chinese version of the Leicester Cough Questionnaire.

Table 3 Multivariate regression analysis of patients with cough after pulmonary resection

\begin{tabular}{|c|c|c|c|c|}
\hline Variables & Wald & OR $(95 \% \mathrm{Cl})$ & B & $P$ value* \\
\hline Subcarinal lymph node dissection & 5.974 & $4.420(1.342-14.554)$ & 1.486 & 0.001 \\
\hline Postoperative acid reflux & 12.45 & $13.550(3.186-57.633)$ & 2.606 & $<0.001$ \\
\hline Duration of anesthesia (exceed 153 minutes) & 6.407 & $0.987(0.978-0.997)$ & -0.013 & 0.011 \\
\hline
\end{tabular}

*, statistically significant $(\mathrm{P}<0.05)$. OR, odds ratios; $\mathrm{Cl}$, confidence interval.

\section{Univariate analysis of CAP}

The univariate analysis of 33 patients in the cough group and 102 patients in the non-cough group showed that factors related to the development of CAP included gender (female), the surgical site (upper right), the resection (lobectomy), subcarinal lymph node dissection, postoperative acid reflux, length of hospitalization (Table 1).

\section{Multivariate regression analysis of $C A P$}

Multivariate logistic regression analysis of the risk factors with statistical significance found in the above univariate analysis was performed. The results showed that the resection (lobectomy) (OR 3.590, 95\% CI: 0.637-20.300, $\mathrm{P}=0.017$ ), subcarinal lymph node dissection (OR 4.420, 95\% CI: $1.342-14.554, \mathrm{P}=0.001)$, postoperative acid reflux (OR 13.550, 95\% CI: 3.186-57.633, $\mathrm{P}<0.001$ ), duration of anesthesia (over 153 minutes, OR 0.987, 95\% CI: $0.978-$ 0.997, $\mathrm{P}=0.011$ ) played a part in CAP development (Table 3).

\section{Discussion}

CAP is a shared complication of thoracic surgery and may develop into a chronic cough. According to the American College of Chest Physicians (ACCP) criteria, the chronic cough should be diagnosed if the cough is the sole or predominant symptom that lasts for longer than eight weeks with no radiographic evidence of lung diseases (21). The persistent postoperative cough troubles a host of patients who undergo postoperative outpatient review. Chronic cough may persist for a long time, affecting patients' postoperative rehabilitation, adding to the psychological burden of patients, and exerting adverse effects on the physical and mental health of patients $(1,5)$. In this prospective study, VAS and CSS were used to test the postoperative cough. Meanwhile, the LCQ-MC survey was conducted before and after surgery to estimate coughing severity and its quality of life impact. The study results proved the resection (lobectomy), subcarinal lymph node dissection, postoperative acid reflux, and duration of anesthesia (over 153 minutes) were risk factors of persistent CAP.

Previously, studies on CAP were rare and centered on thoracotomy and multiport VATS. The application of ERAS theory in thoracic surgery has been deeply rooted $(22,23)$. Surgery is an essential factor in ERAS. The uniportal VATS technique has been increasingly extensively applied in clinical practice in recent years since it can reduce numbers of surgical incisions and patients' pain after surgery, shorten the extubation time, and facilitate faster recovery (14). Therefore, patients are more likely to accept it both physically and psychologically. In our 
study, all patients were operated by uniportal videoassisted thoracoscopic. Our study results proved the resection (lobectomy), subcarinal lymph node dissection, postoperative acid reflux, and duration of anesthesia (over 153 minutes) were risk factors of persistent cough after uniportal video-assisted thoracoscopic pulmonary resection. This is similar to the partial results of two previous studies on cough after thoracotomy or multiport VATS, related studies indicated that postoperative acid reflux, long duration of anesthesia, and subcarinal lymph node dissection are also independent risk factors for CAP. The average operation time took $116.75 \pm 65.08 \mathrm{~min}$, the intra-operative blood loss was $70.52 \pm 80.15 \mathrm{~mL}$, the postoperative chest drainage time was $2.73 \pm 2.03$ days, the average drainage volume was $335.96 \pm 444.19 \mathrm{~mL}$, and the average hospital stay was $10.24 \pm 3.98$ days. All patients were discharged from the hospital after surgery and no one died during the perioperative period. According to the study findings, uniportal thoracoscopy is safe and effective for various types of lung surgery on account of quick hospital stay of patients and rapid postoperative recovery, in line with the concept of ERAS.

At present, a host of tools is available to assess cough, its intensity, frequency, and impact on the quality of life. Both subjective and objective methods should evaluate the postoperative cough. However, studies on an objective evaluation of CAP are scant. The Leicester cough questionnaire (LCQ) in the Guidelines for the Diagnosis and Treatment of Cough in the American Association of Thoracic Physicians is widely used to study adult coughing patients. The LCQ was designed by Birring et al. (24) in 2003, who proved the LCQ could effectively evaluate chronic cough, acute cough, cough after an acute upper respiratory infection, and chronic obstructive pulmonary disease (COPD). Ma et al. (25) first introduced LCQ to China in 2009 and employed it to study the impact of chronic cough on patients' daily life, study and work, and physical and mental health. In 2014, senior researchers translated the LCQ scale into a mandarin version, known as LCQ-MC, and confirmed it was effective in evaluating coughing patients with bronchiectasis (19). Huang et al. (9) further verified the effectiveness, convenience, and reliability of LCQ-MC in evaluating the situation of coughing after surgery o flung cancer. As an effective tool to evaluate the influence of cough on patients' quality of life, the LCQ-MC is more flexible than other coughrelated questionnaires and has practical importance to the clinical evaluation and diagnosis of cough. The LCQ-MC is recommended to appraise the quality of life of patients with a chronic cough by the 2015 Cough Guidelines (20). The Department of Thoracic Surgery of West China Hospital of Sichuan University explored the clinical value of LCQ-MC in estimating the situation of coughing patients after lung surgery. In this study, questionnaire surveys were performed on 121 patients before and after thoracoscopic lung surgery. The results showed that LCQMC was effective in and applicable to the assessment of the cough after thoracoscopic surgery in patients with lung diseases (26). In the present paper, under the guidance of professional thoracic surgery staff, a 5-minute LCQMC survey was conducted to investigate patients' cough conditions before and after the surgery. The procedure was simple. The results indicated that there was no statistical difference in the preoperative LCQ-MC score between the cough group $(16.77 \pm 0.81)$ and the non-cough group $(17.42 \pm 0.98)(\mathrm{P}>0.05)$. The LCQ-MC scores one month and three months after operation $(16.60 \pm 1.02,17.34 \pm 0.70$, respectively) in the cough group were significantly lower than those $(17.64 \pm 0.77,17.89 \pm 0.72$, respectively) in the non-cough group $(\mathrm{P}<0.05)$. The above results suggested that LCQ-MC could objectively and accurately evaluate postoperative cough, and thus guide the treatment of postoperative cough.

Earlier studies have reported the relationship between cough and gender. A study by Lai et al. (12) showed that the gender distribution of chronic cough patients in China is roughly the same. Dicpinigaitis et al. (27) analyzed the cough caused by capsaicin; this cough is closely related to the activation of C-fibers. Their work observed that compared with males, females have a lower capsaicin cough threshold and with more sensitive C-fibers receptors, entirely because of this, females are more likely to cause cough when subjected to related stimuli. A review by Kavalcikova-Bogdanova et al. (13) has also reported that the proportion of patients with chronic cough has a gender difference, with females accounting for the majority. In our study, univariate analysis showed that gender (female) was a risk factor for CAP.

Multivariate logistic regression analysis showed that gender (female) was not an independent risk factor for postoperative cough. These findings contradict a previous study (10). The results of their study showed that female patients are more prone to postoperative cough. Analysis of the reasons may be because our study is a single-center prospective study, and the sample size of the enrolled patients is insufficient. In later studies, it is necessary to 
increase the sample size to corroborate further.

In our study, the subcarinal lymph node dissection was shown to be a significant risk factor of CAP in both univariate and multivariate analyses. The stimulation of C-fibers primarily causes a persistent CAP, and the vagus C-fibers are the most critical cough receptors. Most of the sensory nerve fibers comprise C-fibers and are distributed in the throat, trachea, protuberance, larger bronchial, and small ducts in the lungs. Cough effectors are found both in the mucosal epithelium and walls of the airways. Coughing arises from the physical or chemical stimulation of the throat. For patients with resectable NSCLC, mediastinal lymph node resection enables the correct pathological staging and proper treatment of patients after the operation and prolongs patients' survival time. However, mediastinal lymph node dissection may also increase the difficulty of the operation and the incidence of blood loss, chylous chest, laryngeal nerve injury, and postoperative cough (28). Few efforts were made to research into mediastinal lymph node dissection and CAP, and a few controversies are surrounding them. Sawabata $e t$ al. (2) conducted a cough VAS questionnaire survey of CAP on 240 patients undergoing pulmonary resection (20 cases underwent lobectomy and mediastinal lymph node resection) and analyzed the potential causes of cough. Mediastinal lymph node resection was a factor causing persistent CAP. Also, persistent CAP was believed to be the consequence of the damage to the vagus nerve and its branches during lymph node resection. A double-blind, randomized and controlled clinical trial by Huang et al. (9) divided 100 patients who underwent lobectomy and mediastinal lymph node resection into two groups: the fat-filling group and the non-filling group. Cavities left after lymphadenectomy were filled with fatty tissue autografts in the fat-filling group, while the nonfilling group did not receive any treatment. Their study observed that the cough condition in the fat-filling group was better than that in the non-filling group, showing that filling the gap left after mediastinal lymph node resection with adipose tissue could safely and effectively reduce the occurrence of postoperative cough and improve the quality of life of patients. The observation also proved the association between mediastinal lymph node resection and CAP development from the other side. Given the present and earlier findings, the subcarinal lymph node dissection status might have a slice of clinical influence on CAP in patients who undergo pulmonary resection.

Earlier retrospective studies have reported the relationship between gastroesophageal reflux diseases and the incidence of cough. On the one hand, gastroesophageal reflux diseases start the esophageal-bronchial reaction, activating the vagus nerve from the esophagus to the lungs and resulting in bronchospasm and cough. However, because of the effect of the laryngopharyngeal reflux, gastric coughing spreads through the proximal esophagus and throat to the airway (29). Díaz Vico et al. (30) carried out a retrospective study on 1,149 patients receiving antireflux surgery (41 cases had chronic cough caused by gastroesophageal reflux) and analyzed the clinical efficacy of anti-reflux gastroesophageal fundoplication on patients with gastroesophageal reflux-related cough. All patients completed the same questionnaire at the 6th week and 12th week during the follow-up period. The improvement of their symptoms, cough condition, and quality of life was investigated. The results proved that anti-reflux surgery had a practical treatment effect on intractable cough. They also recommended that correct symptom identification and more appropriate diagnostic tools should be developed to link respiratory symptoms, including cough to gastroesophageal reflux. Sawabata et al. (31) launched an observational and empirical study on 17 patients undergoing lobectomy to explore the relationship between CAP and acid reflux. Thirteen patients developed a persistent cough, and 4 showed no cough symptom, but all patients did not have chronic bronchitis and gastroesophageal reflux diseases. The esophageal hydrogen ion concentration $(\mathrm{PH})$ was watched in all patients for 24 hours, and the severity of the cough was recorded and analyzed using cough VAS. The connection between acid reflux and CAP was observed in some patients. The cough was improved in a slice of patients, but acid reflux still did not relieve one year after surgery. Their study also found that cough was improved in $90 \%$ of patients who used proton pump inhibitors and prokinetic agents, further clarifying that acid reflux after pulmonary resection may lead to a postoperative cough. Postoperative acid reflux was found as a risk factor of CAP in both univariate and multivariate analysis of our study. These findings are in good agreement with the finding of a previous study (2), which established that elevation of the diaphragm or loss of lung volume after pulmonary resection causes gastroesophageal acid reflux, thus resulting in CAP.

Surgical and anesthesia-related factors were proved to affect postoperative cough in earlier studies. Anesthesiarelated factors include anesthesia methods and drugs. DLT intubation general anesthesia is a traditional and commonly used thoracic anesthesia method. Recent studies noticed complications brought by DLT intubation, including airway 
injuries, hoarseness after intubation, sore throat, vocal cord injuries, postoperative cough, among many others (32). To investigate whether spontaneous respiratory anesthesia could reduce the incidence of cough, Chen et al. (11) discussed the impact of different thoracic surgical anesthesia methods on postoperative cough in 1,381 patients who underwent pulmonary resection. All cases were divided into the DLT anesthesia group (group $\mathrm{T}, \mathrm{n}=925$ cases) and spontaneous respiratory anesthesia group (group $\mathrm{S}, \mathrm{n}=456$ cases). Perioperative indicators of the two groups were observed and recorded.

The cough was tested using LCQ-MC and VAS. The results suggested that compared with the DLT general anesthesia, the spontaneous respiratory anesthesia did not interfere with the airway, protected the airway mucosal integrity, reducing the airway inflammatory reaction relieved the postoperative cough, sped up the postoperative rehabilitation, and improved patients' quality of life. A prospective study by Ryu et al. (33) examined whether using a laryngeal mask during total thyroidectomy reduced irritation to pharynx and larynx. They enrolled 76 patients undergoing total thyroidectomy in the study and randomly divided them into the endotracheal tube group (group E) and the laryngeal mask group (group L). The incidence and severity of laryngopharyngeal symptoms, including sore throat, hoarseness, difficulty swallowing and cough, were assessed at 1, 24, and 48 hours after surgery. Their study achieved that compared with traditional tracheal intubation, using a laryngeal mask could reduce the incidence and severity of laryngopharyngeal symptoms. Past works have established that non-intubated thoracoscopic lobectomy is safe and effective for lung cancer patients, capable of reducing the incidence of postoperative sore throat, cough, and complications, and shortening postoperative hospital stay (34). As proven by all the above studies, different anesthesia methods influence the incidence and severity of the postoperative cough. All patients enrolled in our study underwent DLT general anesthesia, and single-lung ventilation during the operation supplied a favorable surgical field, so the effect of different anesthesia methods on postoperative cough was eliminated. The univariate and multivariate analysis results of our study discovered that the duration of anesthesia (over 153 minutes) was an independent risk factor for CAP. Intubation time was the duration from the endotracheal intubation by anesthesiologists to the end of the operation. As found by previous research, airway inflammation plays an essential role in the mechanism of chronic coughing (35).
DLT intubation may cause airway mucosa injuries, airway inflammation, and airway epithelial cells injuries and exposes cough receptors to the shedding of the epithelial cells, causing cough receptors more susceptible to external stimuli. There are many cough receptors in the trachea and throat. The longer the residence time of the DLT in the throat and the trachea are, the more severe the compression that the catheter itself and the airbag cause to trachea mucosa. When the pressure of the catheter balloon exceeds the mucosal capillary pressure, mucosal ischemia leads to tissue edema and inflammation. The accumulation of acidic substances and the change of airway $\mathrm{PH}$ value during inflammation can stimulate $\mathrm{C}$ nerve fibers, excite the nerve center, cause airway neuroinflammation, and induce bronchial contraction (36). All the above factors will increase the incidence of CAP.

As reported in past research, changes in the structure and function of residual lungs and bronchus trachealis after lung resection may lead to postoperative complications, including dyspnea and cough. Ueda et al. (37) concluded that increased airway resistance during exhalation caused by bronchial kink might cause lung tissue structure and function mismatched after lobectomy. The increase in airway resistance may also prolong air retention or increase functional residual volume, thus significantly reducing postoperative lung function volume and ventilation volume. Therefore, the exertional dyspnea and postoperative cough occur, exerting adverse effects on the quality of life of patients. CT imaging and computational fluid dynamics (CFD) were used by $\mathrm{Gu}$ et al. (38) to analyze the relationship of changes in the structure and function of the tracheobronchial tree after the left upper lobe (LUL) resection with postoperative cough and dyspnea. They quantitatively measured the structural changes of the tracheobronchial tree in 18 patients who had undergone LUL resection, including the angle between the trachea and bronchus, the surface area and volume of the tree, and the cross-sectional area of the left lower lobe bronchus. Apart from this, the CFD technique was used to measure the wall pressure, gas velocity, pressure drop, lobar flow rate, and local flow features. Their findings revealed that the structure and function of the tracheobronchial tree experienced a series of changes after LUL resection, including a reduced angle between a left main bronchus and left main bronchus, the sigmoidal distortion of the left main bronchus, and left lower lobe bronchus stenosis. These structural changes result in abnormal increases in the inflow velocity, wall pressure, wall shear stress, vortex probability, and pressure drop, causing damage to the airways and 
mucous membranes and bringing about inflammatory reactions in the long term.

Postoperative dyspnea and postoperative cough develop. A retrospective study by Seok et al. (39) investigated the effect of bronchial deviation degree on postoperative lung function in 99 lung cancer patients after upper lobectomy. Among these patients, 50 received LUL resection, and 49 underwent right upper lobe resection (RUL). Chest CT measured the angle between the main bronchus and other bronchi in the coronal position. Three and 12 months after surgery, how much dyspnea and pulmonary function were examined, and chest radiography and chest CT were performed. The results showed that although it was merely a physiological change, the increased bronchial angle after upper lobectomy might do serious harm to the lung function of patients, causing postoperative shortness of breath, dyspnea, and cough. The resection (lobectomy) was found as an independent risk factor for CAP in our study. Under the same conditions, lobectomy causes more apparent changes to the structure and function than sublobar resection (including wedge resection and segmentectomy) - the pulmonary lobe resection results in a decrease in lung volume of the affected side. The upward displacement of the remaining lungs and diaphragm can lead to residual bronchial malformations, and even bronchi distortion or obstruction in more severe cases (40). The airflow dynamics and airway sensitivity of the bronchus trachealis are affected by the structural variations, and longterm structural changes give rise to postoperative cough. Also, the univariate analysis of this study showed that right upper lung surgery was a risk factor for CAP, but the results of multivariate analysis showed that right upper lung surgery was not an independent risk factor for postoperative cough. This is contrary to an earlier study that analyzed clinical data of 650 cases of lung cancer underwent thoracoscopic lobectomy. The reason for the analysis may be that RUL surgery in our study was performed on 45 patients, in which lobectomy on 22 patients and sublobar resection on 23 patients. Compared with lobectomy, losing lung volume is less in sublobar resection. There are more minor changes in the structure and physiology of the residual lung, which have a smaller effect on cough.

Our study also has a slice of limitations. First, it is a single-center prospective study with a small sample size, so there may be statistical differences in the assessment of individual variables, in the later research, the sample size will be expanded to try to avoid the bias. Second, we focused on the causes of postoperative cough but ignored the mechanism of these causes and the intervention measures for postoperative cough. The future study should put more emphasis on the mechanism and treatment measures of postoperative cough and lay the foundation for perfecting the lung operation plan to decrease the occurrence of CAP.

\section{Conclusions}

In conclusion, uniportal VATS is possible for several types of lung surgery and conducive to enriching the ERAS concept. CAP is a more shared complication. This study proves subcarinal lymph node dissection, postoperative acid reflux, duration of anesthesia (over 153 minutes), and the type of resection (lobectomy) are risk factors for persistent CAP.

\section{Acknowledgments}

Funding: This work was supported by the grants from National Natural Science Foundation of China (81873417).

\section{Footnote}

Reporting Checklist: The authors have completed the STROBE reporting checklist. Available at http://dx.doi. org/10.21037/jtd-20-2652

Data Sharing Statement: Available at http://dx.doi. org/10.21037/jtd-20-2652

Conflicts of Interest: All authors have completed the ICMJE uniform disclosure form (available at http://dx.doi. org/10.21037/jtd-20-2652). The authors have no conflicts of interest to declare.

Ethical Statement: The authors are accountable for all aspects of the work in ensuring that questions related to the accuracy or integrity of any part of the work are appropriately investigated and resolved. The study protocol was approved by the Ethics Committee of The First Affiliated Hospital of Soochow University, Suzhou, China (NO.2019-113). All patients have signed the written informed consent before the operation. The study was conducted in accordance with the Declaration of Helsinki (as revised in 2013).

Open Access Statement: This is an Open Access article distributed in accordance with the Creative Commons 
Attribution-NonCommercial-NoDerivs 4.0 International License (CC BY-NC-ND 4.0), which permits the noncommercial replication and distribution of the article with the strict proviso that no changes or edits are made and the original work is properly cited (including links to both the formal publication through the relevant DOI and the license). See: https://creativecommons.org/licenses/by-nc-nd/4.0/.

\section{References}

1. Morice AH, Jakes AD, Faruqi S, et al. A worldwide survey of chronic cough: a manifestation of enhanced somatosensory response. Eur Respir J 2014;44:1149-55.

2. Sawabata N, Maeda H, Takeda S, et al. Persistent cough following pulmonary resection: observational and empiric study of possible causes. Ann Thorac Surg 2005;79:289-93.

3. Zhu M, Wu C, Wu Z, et al. Acute exacerbation of interstitial lung disease after radical surgery for lung cancer: a case report. Ann Transl Med 2019;7:182.

4. Yang P, Cheville AL, Wampfler JA, et al. Quality of life and symptom burden among long-term lung cancer survivors. J Thorac Oncol 2012;7:64-70.

5. Young EC, Smith JA. Quality of life in patients with chronic cough. Ther Adv Respir Dis 2010;4:49-55.

6. Dicpinigaitis PV. Clinical perspective - cough: an unmet need. Curr Opin Pharmacol 2015;22:24-8.

7. Polverino M, Polverino F, Fasolino $M$, et al. Anatomy and neuro-pathophysiology of the cough reflex arc. Multidiscip Respir Med 2012;7:5.

8. Canning BJ, Chang AB, Bolser DC, et al. Anatomy and neurophysiology of cough: CHEST Guideline and Expert Panel report. Chest 2014;146:1633-48.

9. Huang J, Luo Q, Tan Q, et al. Evaluation of the surgical fat-filling procedure in the treatment of refractory cough after systematic mediastinal lymphadenectomy in patients with right lung cancer. J Surg Res 2014;187:490-5.

10. Lin R, Che G. Risk factors of cough in non-small cell lung cancer patients after video-assisted thoracoscopic surgery. J Thorac Dis 2018;10:5368-75.

11. Chen Z, Dong Q, Liang L. Effect of different thoracic anesthesia on postoperative cough. J Thorac Dis 2018;10:3539.

12. Lai K, Long L, Yi F, et al. Age and Sex Distribution of Chinese Chronic Cough Patients and Their Relationship With Capsaicin Cough Sensitivity. Allergy Asthma Immunol Res 2019;11:871-84.

13. Kavalcikova-Bogdanova N, Buday T, Plevkova J, et al. Chronic cough as a female gender issue. Respiratory
Contagion. Springer; 2015:69-78.

14. McElnay PJ, Molyneux M, Krishnadas R, et al. Pain and recovery are comparable after either uniportal or multiport video-assisted thoracoscopic lobectomy: an observation study. Eur J Cardiothorac Surg 2015;47:912-5.

15. Liu X, Chen X, Shen Y, et al. Learning curve for uniportal video-assisted thoracoscopic surgery lobectomyresults from 120 consecutive patients. J Thorac Dis 2018;10:5100-7.

16. Mountain CF, Dresler CM. Regional lymph node classification for lung cancer staging. Chest 1997;111:1718-23.

17. Chinese guidelines for diagnosis and treatment of primary lung cancer 2018 (English version). Chin J Cancer Res 2019;31:1-28.

18. Enrique R, Marta B, Javier Z, et al. Is the reflux disease questionnaire useful for identifying GERD according to the Montreal definition? BMC Gastroenterol 2014;14:17.

19. Gao Y, Guan W, Xu G, et al. Validation of the Mandarin Chinese version of the Leicester Cough Questionnaire in bronchiectasis. Int J Tuberc Lung Dis 2014;18:1431-7.

20. Lai K. Chinese National Guidelines on Diagnosis and Management of Cough: consensus and controversy. J Thorac Dis 2014;6:S683-8.

21. Pratter MR, Brightling CE, Boulet LP, et al. An empiric integrative approach to the management of cough: ACCP evidence-based clinical practice guidelines. Chest 2006;129:222S-231S.

22. Gao K, Yu PM, Su JH, et al. Cardiopulmonary exercise testing screening and pre-operative pulmonary rehabilitation reduce postoperative complications and improve fast-track recovery after lung cancer surgery: A study for 342 cases. Thorac Cancer 2015;6:443-9.

23. Li S, Che G, Shen C, et al. Current situation and consideration on the enhanced recovery protocols in lung cancer surgery. J Thorac Dis 2018;10:S3855-8.

24. Birring S, Prudon B, Carr A, et al. Development of a symptom specific health status measure for patients with chronic cough: Leicester Cough Questionnaire (LCQ). Thorax 2003;58:339-43.

25. Ma W, Yu L, Wang Y, et al. Changes in health-related quality of life and clinical implications in Chinese patients with chronic cough. Cough 2009;5:7.

26. $\mathrm{Xu} Z$, Lin R, Che G, et al. Validation of the Mandarin Chinese Version of the Leicester Cough Questionnaire in Patients Undergoing Lung Resection for Patients with Lung Disease. Zhongguo Fei Ai Za Zhi 2017;20:389-94.

27. Dicpinigaitis PV, Rauf K. The influence of gender on 
cough reflex sensitivity. Chest 1998;113:1319-21.

28. Darling GE, Allen MS, Decker PA, et al. Randomized trial of mediastinal lymph node sampling versus complete lymphadenectomy during pulmonary resection in the patient with N0 or N1 (less than hilar) non-small cell carcinoma: results of the American College of Surgery Oncology Group Z0030 Trial. J Thorac Cardiovasc Surg 2011;141:662-70.

29. Esposito C, Saxena A, Irtan S, et al. Laparoscopic Nissen Fundoplication: An Excellent Treatment of GERDRelated Respiratory Symptoms in Children-Results of a Multicentric Study. J Laparoendosc Adv Surg Tech A 2018;28:1023-8.

30. Díaz Vico T, Elli EF. Clinical outcomes of gastroesophageal reflux disease-related chronic cough following antireflux fundoplication. Esophagus 2020;17:92-8.

31. Sawabata N, Takeda S, Tokunaga T, et al. Acid regurgitation associated with persistent cough after pulmonary resection: an observational study. Cough 2006;2:9.

32. Wu CY, Chen JS, Lin YS, et al. Feasibility and safety of nonintubated thoracoscopic lobectomy for geriatric lung cancer patients. Ann Thorac Surg 2013;95:405-11.

33. Ryu JH, Yom CK, Park DJ, et al. Prospective randomized controlled trial on the use of flexible reinforced laryngeal mask airway (LMA) during total thyroidectomy: effects on postoperative laryngopharyngeal symptoms. World J Surg 2014;38:378-84.

34. Hung MH, Chan KC, Liu YJ, et al. Nonintubated thoracoscopic lobectomy for lung cancer using epidural anesthesia and intercostal blockade: a retrospective cohort study of 238 cases. Medicine (Baltimore) 2015;94:e727.

35. Ricciardolo FL. Mechanisms of citric acid-induced bronchoconstriction. Am J Med 2001;111 Suppl 8A:18S-24S.

36. Widdicombe J. Airway receptors. Respir Physiol 2001;125:3-15.

37. Ueda K, Tanaka T, Hayashi M, et al. Clinical ramifications of bronchial kink after upper lobectomy. Ann Thorac Surg 2012;93:259-65.

38. Gu Q, Qi S, Yue Y, et al. Structural and functional alterations of the tracheobronchial tree after left upper pulmonary lobectomy for lung cancer. Biomed Eng Online 2019;18:105.

39. Seok Y, Cho S, Lee JY, et al. The effect of postoperative change in bronchial angle on postoperative pulmonary function after upper lobectomy in lung cancer patients. Interact Cardiovasc Thorac Surg 2014;18:183-8.

40. Ueda K, Hayashi M, Tanaka N, et al. Long-term pulmonary function after major lung resection. Gen Thorac Cardiovasc Surg 2014;62:24-30.
Cite this article as: Pan LY, Peng LP, Xu C, Ding C, Chen J, Wang WY, Zhu XY, Zhao J, Li C. Predictive factors of cough after uniportal video-assisted thoracoscopic pulmonary resection. J Thorac Dis 2020;12(10):5958-5969. doi: 10.21037/ jtd-20-2652 